\title{
Surgical Management of Proximal Tibial Physeal Fracture in a Dog
}

\author{
Tarunbir Singh*, Priyanka, Pallavi Verma, \\ Jitender Mohindroo and Rahul Kumar Udehiya \\ Department of Veterinary Surgery and Radiology, Guru Angad Dev Veterinary and Animal \\ Sciences University, Ludhiana, Punjab, India \\ *Corresponding author
}

Keywords

Tibia, Physeal fracture, Cross pinning, T-plate, Lag screw

Article Info

Accepted: 20 January 2021 Available Online: 10 February 2021
A 6 month old male intact French mastiff was presented for left pelvic limb lameness 2 days after the dog had been hit by a motor vehicle. Medio-lateral and cranio-caudal radiographs of the left stifle revealed a Salter-Harris type 2 fracture of the proximal tibial physis with segmental fracture of fibula. A medial surgical approach allowed reduction and stabilisation of proximal tibial physeal fracture with two $1.4 \mathrm{~mm}$ crossed kirschner wires, a 3.5-mm lag screw and a 6 holed $3.5 \mathrm{~mm}$ T-plate. Modified Robert Jones bandaging was done to reduce stifle motion. Satisfactory fracture reduction was confirmed in immediate post-operative radiographs. Radiographic re-evaluation revealed periosteal reaction at fracture site at two weeks and healed tibial physis and fibular fracture with remodelling of periosteal reaction and stable implant at 3 months postoperatively. The owner reported the dog had resumed normal activities and regained full limb usage without showing any sign of lameness. Physeal fracture was reduced using a combination of pin and plate, suggesting that surgical treatment of these challenging and uncommon fractures may be more appropriately achieved by this technique.

\section{Introduction}

Fractures of the proximal tibial physis are relatively uncommon and account for only 3.7 per cent of all physeal fractures in small animals (Marretta and Schrader 1983).There are two centres of ossification in the proximal tibia of dogs namely the proximal tibial epiphysis and the tibial tuberosity apophysis. The proximal tibialphysis closes at an average age of 10.7 months and the physis of the tibial tuberosity closes at an average of 8.3 months (Manley et al., 1990). It is hypothesised that Contraction of the quadriceps muscle group against stifle flexion results in a proximal distractive force on the tibial tuberosity which leads to this type of fracture (Pratt, 2001). Fibular fractures are occasionally associated with separation of the proximal tibial physis and avulsion of tibial tuberosity. Various conservative and internal method of stabilisation of these types of fractures have been documented (Clements et al., 2003). It has been suggested that if the avulsion of the tibial tuberosity has a displacement of less than $3 \mathrm{~mm}$, it can be treated conservatively (Withrow et al., 1976). However, it is recommended that all avulsions of the 
tuberosity combined with a proximal tibialphyseal separation should be reduced to prevent any change in the tibial plateau inclination angle.

\section{Case description}

A six-month-old male French mastiff was presented with a non-weight-bearing on left hind limb after being hit by a motor vehicle. Medio-lateral and cranio-caudal radiograph of affected limb revealed Salter Harris type 2 fracture of the left proximal tibialphysis, with a large lateral oblique metaphyseal component and segmental fracture of fibula (Fig. 1). Marked medial displacement of the tibial diaphysis was noted. There was moderate swelling and pain over left stifle region.

\section{Treatment}

Dog was premedicated with a combination of Inj. Butorphanol @ $0.2 \mathrm{mg} / \mathrm{kg}$ b.wt., Inj. Acepromazine @ $0.05 \mathrm{mg} / \mathrm{kg}$ b.wt. and Inj. Glycopyrrolate @ $0.01 \quad \mathrm{mg} / \mathrm{kg} \quad$ b.wt. intramuscularly. Anaesthesia was induced with Inj. Propofol @ 4 mg/kg b.wt. I.V. and then maintained on 1-2\% Isoflurane in oxygen through cuffed endotracheal tube connected to the circle system of small animal anaesthetic machine. Normal saline solution (NSS) was administered intravenously as continuous infusion throughout the operative period till the recovery from anaesthesia. The site was aseptically prepared. A medial surgical approach to the right proximal tibia (Piermattei, 1993) facilitated exposure, but not reduction of the proximal tibial physis. Transection of the distal soft tissue attachments of the tibial tuberosity to the proximal tibia allowed reduction of the proximal tibial epiphysis, which was stabilised by cross pinning with two1.4-mm Kirschner (K) wires, a lag screw and 6hole3.5-mm T-plate. Proximal fragment was first stabilised with $1.4 \mathrm{~mm}$ kirschner wire which was inserted from cranio-medial side between collateral ligament and tibial tuberosity and second wire inserted from cranio-lateral approach (Fig. 5). A lag screw was also placed to stabilise the proximal epiphyseal fracture fragment, then a 6 hole Tplate was placed medially by giving small incision over medial side (Fig. 6). The muscles, subcutaneous and skin were sutured in routine manner.

\section{Results and Discussion}

Immediate post-operative radiograph revealed satisfactory fracture reduction and kirschner wire, lag screw and T-plate placement (Fig. 2). Modified Robert Jones bandaging was done till the date of suture removal to provide external support and to reduce stifle motion. Re-evaluation two weeks post-operatively revealed the dog to be moderately weight bearing on operated limb. Radiographs revealed new bone formation at fracture site and stable implants (Fig. 3). Complete bone union and closure of tibial physis were noted on 3 month post-operative radiographs. Radiography at 3 months post-operatively revealed healed tibial physis and fibular fracture with remodelling of periosteal reaction and stable implant (Fig. 4). Dog was excellently weight bearing on operated limb (Fig. 8) and no visible difference between pelvic limb was noted (Fig. 7). Telephonic follow-up at 5 months post-operatively, the owner reported that the dog had resumed normal activities without any sign of lameness.

Fractures of the proximal tibialphysis are not very common in dogs (Marretta and Schrader 1983). Minimally displaced fractures that can be reduced in a closed fashion can be successfully managed conservatively with coaptation (Clements et al., 2003). Mildly or moderately displaced fractures can be reduced 
by means of open reduction, are typically stabilised with divergent interfragmentary Kirschner wires (Clements et al., 2009). This case was managed using Arbeitsgemeinschaft fur Osteosynthesefragen/ Association for the Study of Internal Fixation (AO/ASIF) principles, namely, rigid internal fixation, accurate anatomical reduction, the preservation of blood supply and early postoperative mobilisation. Intra-operatively it was difficult to position the $\mathrm{T}$-plate for stabilising proximal tibial epiphysis.

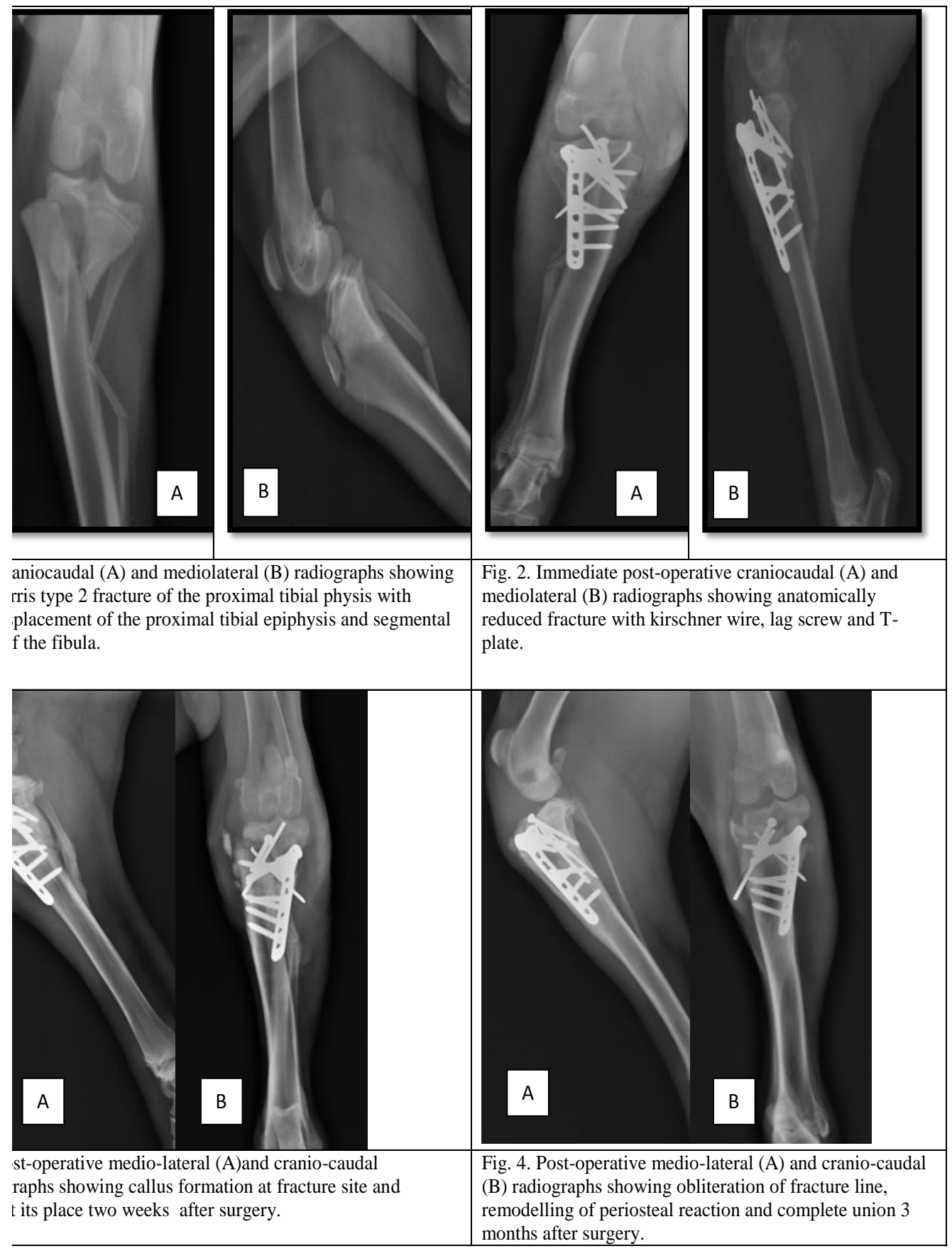



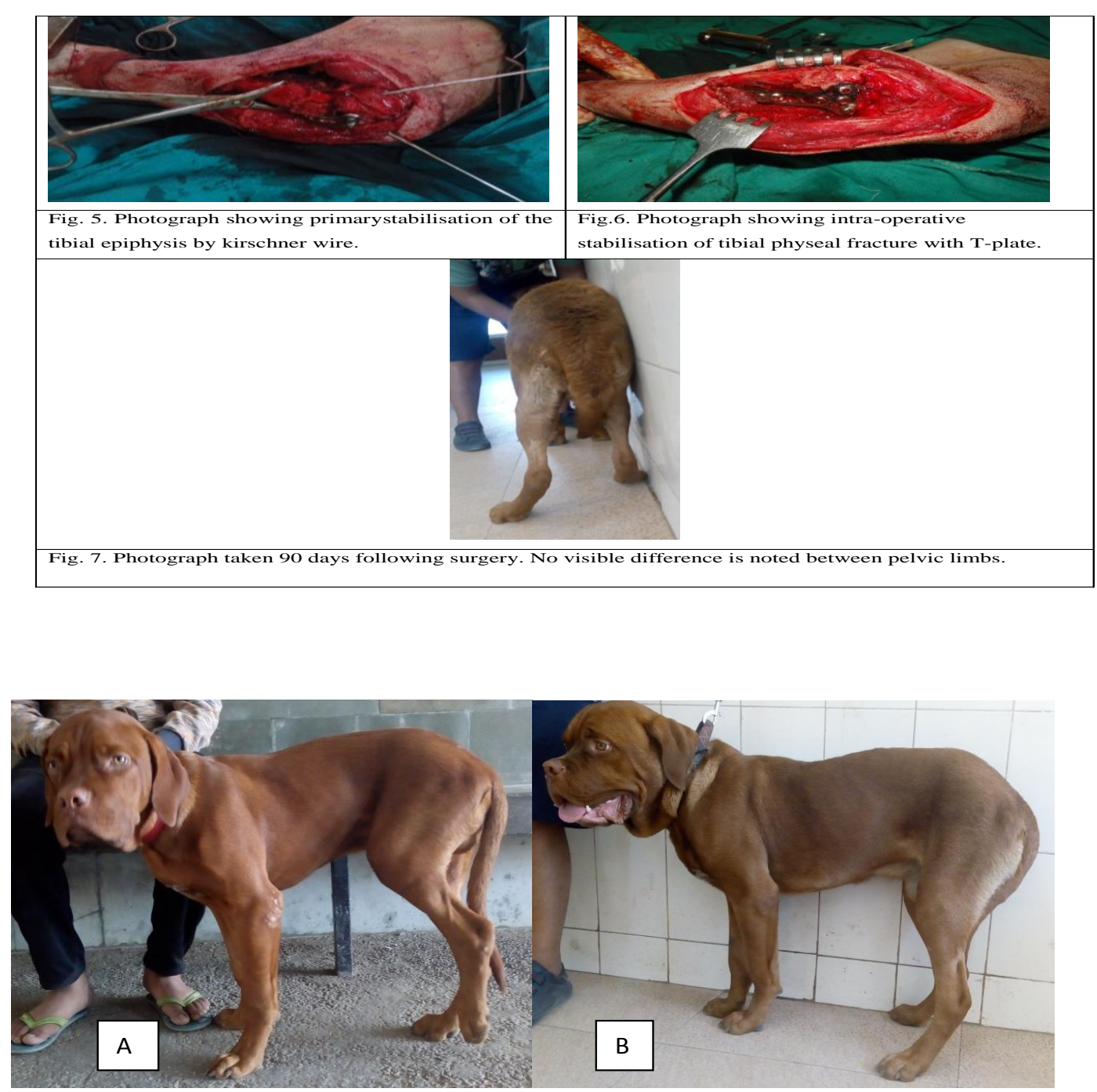

Fig. 8: Pre-operative (A) and Post-operative (B) images of dog. In image B complete weight bearing on operated left hind limb 3 months post operatively.

This depicts the challenging alignment of the fracture and the difficulties in positioning adequate internal fixation in the proximal tibial epiphysis. The difficulty encountered in reduction of this fracture was due to mild overriding of the fracture segments. Intraoperative application of kirschner wire as well as lag screw simplified the placement of $\mathrm{T}$ plate. This technique facilitated rapid reduction and rigid stability intra as well as post-operatively. For open reduction this technique could prove advantageous in stabilising proximal tibial physeal fracture. In conclusion, the laterally displaced proximal tibialphyseal fractures can be managed successfully with kirschner wire, lag screw and T plate.

\section{Acknowledgement}

Financial sanction from Indian council of Agricultural research under ICAR 22 all India network programme on diagnostic imaging and management of surgical conditions in animals is hereby acknowledged. 


\section{References}

Marretta, S.M. and Schrader, S.C. (1983). Physeal injuries in the dog: a review of 135 cases. Journal of the American Veterinary Medical Association 182(7): 708. Manley, PA., Henry, W.B., Wilson, J.W. and Whittick, W.G. (1990). Diseases of the epiphyses. Canine Orthopedics. Lea Febiger, Philadelphia, Pp. 585-619.

Pratt, J.N. (2001). Avulsion of the tibial tuberosity with separation of the proximal tibial physis in seven dogs. Veterinary Record149: 352-356

Clements, D.N., Gemmill, T., Corr, S.A., Bennett, D. and Carmichael, S. (2003). Fracture of the proximal tibial epiphysis and tuberosity in 10 dogs. Journal of small animal practice 44(8): 355-358. Withrow, S., DeAngelis, M., Arnoczky, S. and Rosen, H. (1976). Treatment of fractures of the tibial tuberosity in the dog. Journal of the American Veterinary Medical Association 168(2): 122-124.

Piermattei, D.L. (1993). Approach to the stifle through a medial incision. In: Atlas of Orthopaedic Surgical Procedures in the Dog and Cat. Ed D. L. Piermattei. W. B. Saunders, Philadelphia, PA, USA 278281

Clements, D.N., Clarke, S.P. and Mosley, J.R. (2009) Management of laterally displaced proximal tibial physeal fractures in three dogs. Journal of Small Animal Practice, 50: 662-666.

\section{How to cite this article:}

Tarunbir Singh, Priyanka, Pallavi Verma, Jitender Mohindroo and Rahul Kumar Udehiya. 2021. Surgical Management of Proximal Tibial Physeal Fracture in a Dog. Int.J.Curr.Microbiol.App.Sci. 10(02): 2392-2396. doi: https://doi.org/10.20546/ijcmas.2021.1002.284 